\title{
Developing a genome-wide selection model for genetic improvement of residual feed intake and carcass merit in a beef cattle breeding program
}

\author{
ZENG ZhiYao ${ }^{1,2}$, TANG GuoQing ${ }^{1}$, MA JiDeng ${ }^{1}$, PLASTOW Graham ${ }^{2}$, MOORE Stephen ${ }^{2}$, \\ LAI SongJia $^{1 *}$, LI XueWei ${ }^{1 *} \&$ WANG ZhiQuan ${ }^{2,3 *}$ \\ ${ }^{1}$ College of Animal Science and Technology, Sichuan Agricultural University, Yaan 625014, China; \\ ${ }^{2}$ Department of Agricultural, Food and Nutritional Science, University of Alberta, Edmonton, AB T6G 2C8, Canada; \\ ${ }^{3}$ College of Animal Science and Technology, Heilongjiang August First Land Reclamation University, Daqing 163319, China
}

Received April 25, 2012; accepted May 28, 2012

\begin{abstract}
Residual feed intake (RFI) and carcass merit (CM) are both complex traits emerging as critical targets for beef genetic improvement. RFI and CM traits are difficult and expensive to measure and genetic improvement for these traits through traditional selection methods is not very effective. Therefore, genome-wide selection using DNA markers may be a potential alternative for genetic improvement of these traits. In this study, the efficiency of a genome-wide selection model for genetic improvement of RFI and CM was assessed. The Illumina Bovine50K bead chip was used to genotype 922 beef cattle from the Kinsella Beef Research Ranch of the University of Alberta. A Bayes model and multiple marker regression using a stepwise method were used to conduct the association test. The number of significant SNP markers for carcass weight (CWT), carcass back fat (BF), carcass rib eye area (REA), carcass grade fat (GDF), lean meat yield (LMY), and residual feed intake (RFI) were 75, 54, 67, 57, 44 and 50, respectively. Bi-variate analysis of marker scores and phenotypes for all traits were made using DMU Software. The genetic parameter for each trait was estimated. The genetic correlations of marker score and phenotype for CWT, BF, REA, GDF, LMY and RFI were $0.75,0.69,0.87,0.77,0.78$, and 0.85 , respectively. The average prediction accuracies of phenotypic EBV for the six traits were increased by $0.05,0.16,0.24,0.23,0.17$ and 0.19 , respectively. The results of this study indicated that the two-trait marker-assisted evaluation model used was a suitable alternative of genetic evaluation for these traits in beef cattle.
\end{abstract}

marker-assisted selection, beef cattle, marker score, carcass traits, RFI

Citation: Zeng Z Y, Tang G Q, Ma J D, et al. Developing a genome-wide selection model for genetic improvement of residual feed intake and carcass merit in a beef cattle breeding program. Chin Sci Bull, 2012, 57: 2741-2746, doi: 10.1007/s11434-012-5325-6

Beef breeders need new methods to optimize selection for all economically important traits. For example, in order to improve the efficiency of quality beef they need to select for cattle that have lower residual feed intake (RFI) and desirable carcass merit (CM). RFI is defined as the difference between an animal's actual feed intake and its expected intake for maintenance and production [1]. Selection for RFI could save inputs and maximize profits by decreasing feed costs. CM is used in assessing carcass traits including carcass weight (CWT), carcass back fat $(\mathrm{BF})$, carcass rib eye area (REA), carcass grade fat (GDF), and lean meat yield (LMY).

The phenotypes of RFI and CM are expensive and difficult to measure, which makes them very suitable for markerassisted selection (MAS) [2] or genomic selection (GS) [3]. GS is the selection of animals for breeding based on estimated breeding values, which are calculated from the joint effects of genetic markers covering the entire genome. GS is

*Corresponding authors (email: 1sj5781@263.net; xuewei.li@sicau.edu.cn; zhiquan@ualberta.ca) 
expected to double the rate of genetic improvement per year in many livestock systems [4] and has been successfully applied in dairy cattle genetic evaluation programs $[5,6]$ in many countries. However, GS in beef cattle is not as successful as that in dairy cattle to date due to small reference population and family size, which restricts the evaluation accuracy of marker effects, compared with dairy. Therefore, a more efficient use of available molecular information in the genetic evaluation of beef cattle requires incorporating the molecular marker score in genome-wide MAS. Kachman [7] proposed a new two-trait marker-assisted evaluation model (MABLUP, two-trait model, including marker score and phenotype) to incorporate marker information, which treated the marker score as a correlated trait of the phenotype under evaluation, and improved the estimation accuracy of the phenotypic EBV using the marker score information in bi-variable model evaluation. MacNeil et al. [8] applied this method to assess the utility of molecular breeding value (MBV, marker score of genome) in predicting the breeding value for marbling score. Subsequently, Tang et al. [9] developed marker assisted selection models for evaluating growth traits by this method. However, both of the above studies used panels with a small number of markers that limited the genetic correlation between phenotype and marker score. The objective of this paper, therefore, was to develop the genome-wide two-trait MABLUP selection model for evaluating RFI and CM traits using high-density SNP (the Bovine50 Bead Chip) genotype data resulting from animals raised at the Kinsella Beef Research Ranch of the University of Alberta.

\section{Materials and methods}

\subsection{Phenotype and genotype data}

A total of 922 steers from the Kinsella Research Ranch of the University of Alberta were used in this study. The animals were managed and cared for according to the guidelines of the Canadian Council of Animal Care [10]. The composition of this population was produced by crossing between Angus, Charolais or University of Alberta hybrid bulls and a hybrid dam line. The measurements and ultrasound traits were obtained as part of the phenotypic data collection during the feedlot tests that were conducted at the Kinsella Ranch from 2003 to 2008 with two batches of steers tested per year. The CM traits were collected in the abattoir, which was described by Nkrumah et al. [11]. CWT was measured as a summation of the left and right halves of each carcass. BF was the fat thickness measured over the ribeye muscle at the 12 th rib. REA was measured on the cross section of the muscle between the 12th and 13th ribs. GDF was measured at the 12th-13th rib. LMY, an estimation of the saleable meat, was estimated using the following equation: Lean meat yield $(\%)=57.96+(0.202 \times$ L. thoracis area $\left(\mathrm{cm}^{2}\right)-(0.027 \times$ warm carcass weight $(\mathrm{kg}))-$
$(0.703 \times$ average backfat thickness $(\mathrm{mm}))$ as described by Basarab et al. [12]. The residuals from the equation (shown below) were assigned as residual feed intake (RFI), $Y_{j}=\beta_{0}$ $+\beta_{1} \mathrm{ADG}_{j}+\beta_{2} \mathrm{MWT}_{j}+\beta_{3} \mathrm{UBF}_{j}+e_{j}$, where, for each animal, $Y_{j}$ is the standardized DMI, $\beta_{0}$ is the regression intercept, $\beta_{1}$ is the average daily gain (ADG) regression coefficient, $\beta_{2}$ is the metabolic body weight (MWT, which is the mid-test body weight raise the power of 0.75 ) regression coefficient, $\beta_{3}$ is the ultrasound backfat (UBF) regression coefficient, and $e_{j}$ indicates the residuals (RFI). Nine hundred and twenty-two beef steers from this herd were genotyped using the Illumina Bovine 50K Bead Chip for the purposes of QTL mapping and candidate gene search in the previous research projects. The number of phenotype records for CWT, BF, REA, GDF, LMY, and RFI were 836, 836, 836, 836, 836, and 852 , respectively. The descriptive statistics for these traits are summarized in Table 1.

\subsection{The filtering of SNP markers}

The genotyping data of these animals were first analyzed by a quality examination to remove the disqualified markers (Minor allele frequency $<0.05$ and Hardy-Weinberg chisquare value $>600$ ) to minimize the probability of false association and avoid any probability of genotyping errors based on the method described by Hayes et al. [13]. After the quality examination, a total of 40809 SNP markers were retained in the analysis.

\subsection{The association test}

Associations between each trait with all SNP markers were assessed using the GENSEL software developed by Iowa State University [14] with the model below:

$$
y=W f+\sum_{j=1}^{n} X_{j} g_{j}+e,
$$

where $y$ is observation vector; $f$ is the fixed effects vector including contemporary group of test year and test group combinations, sire breed for all CM and RFI traits, and plus an additional covariate of slaughter age for all CM traits; $W$ is the design matrices that relate $f$ to their corresponding records in $y ; g_{j}$ is the allele substitution effect of the $j$ th

Table 1 The descriptive statistics for carcass weight (CWT), carcass back fat $(B F)$, carcass rib eye area (REA), carcass grade fat (GDF), lean meat yield (LMY), and residual feed intake (RFI)

\begin{tabular}{lccccc}
\hline Trait & Phenotype & Mean & Min & Max & Std Dev \\
\hline CWT & 836 & 317.98 & 207.2 & 453.2 & 28.76 \\
BF & 836 & 12.45 & 2.67 & 26.67 & 3.98 \\
REA & 836 & 82.94 & 53 & 113 & 8.39 \\
GDF & 836 & 11.03 & 2 & 26 & 3.95 \\
LMY & 836 & 57.38 & 44.67 & 66.18 & 3.66 \\
RFI & 852 & -0.0037 & -2.82 & 3.23 & 0.91 \\
\hline
\end{tabular}


SNP marker; $X_{j}$ is the design matrix to allocate records to the $j$ th marker; $n$ is the number of SNP markers that are included in the model; $e$ is the residual error vector and $e \sim N$ $\left(0, I \sigma_{e}^{2}\right)$, where $I$ is an identity matrix and $\sigma_{e}^{2}$ is the residual variance.

The module of BayesC was first used to estimate the variance component. Secondly, the module of BayesCpi was used to evaluate a reasonable $\pi$ value (marker effect $=0$ with probability $\pi$ ). Then, the estimated $\pi$ value was used in the BayesB module (1) to estimate the effect and variance for each marker.

The top 200 markers resulting from BayesB model according to the size of variance were selected and fitted into a linear model (2) to re-filter the markers based on multiple markers regression using a stepwise method.

$$
y=W f+\sum_{j=1}^{200} X_{j} g_{j}+e .
$$

\subsection{The calculation of marker score}

Significant markers $(P<0.10)$ from the linear model (model (2)) were chosen for the linear model below to calculate marker score for MAS.

$$
M S_{i}=\sum_{j=1}^{n} X_{i j} g_{j},
$$

where $M S_{i}$ is the marker score for the $i$ th animal, $g_{j}$ is the allele substitution effect of the $j$ th SNP marker estimated from the linear model, $X_{i j}$ is the number of copies of a specific marker allele of the $j$ th SNP marker for the $i$ th animal, $n$ is the number of significant SNP marker in MAS.

\subsection{The two-trait marker-assisted evaluation model development}

A bi-variate residual maximum likelihood (REML) method was used to estimate the genetic variance and covariance of marker score and phenotype using the DMU software developed by University of Aarhus [15] and the two-trait marker-assisted evaluation models [7,16] were developed for the RFI and five CM traits with the following models:

(i) $\mathrm{CWT}_{\mathrm{P}}=$ mean $+\mathrm{CGP}+\mathrm{SB}+\mathrm{SAGE}($ covariate $)+$ Animal + Residual;

$\mathrm{CWT}_{\mathrm{M}}=$ mean + Animal + Residual.

(ii) $\mathrm{BF}_{\mathrm{P}}=$ mean $+\mathrm{CGP}+\mathrm{SB}+\mathrm{SAGE}($ covariate $)+\mathrm{An}-$ imal + Residual;

$\mathrm{BF}_{\mathrm{M}}=$ mean + Animal + Residual .

(iii) $\mathrm{REA}_{\mathrm{P}}=$ mean $+\mathrm{CGP}+\mathrm{SB}+\mathrm{SAGE}($ covariate $)+$ Animal + Residual;

$\mathrm{REA}_{\mathrm{M}}=$ mean + Animal + Residual.

(iv) $\mathrm{GDF}_{\mathrm{P}}=$ mean $+\mathrm{CGP}+\mathrm{SB}+\mathrm{SAGE}($ covariate $)+$ Animal + Residual;

$\mathrm{GDF}_{\mathrm{M}}=$ mean + Animal + Residual.

(v) $\mathrm{LMY}_{\mathrm{P}}=$ mean $+\mathrm{CGP}+\mathrm{SB}+\mathrm{SAGE}($ covariate $)+$ Animal + Residual;
$\mathrm{LMY}_{\mathrm{M}}=$ mean + Animal + Residual.

(vi) $\mathrm{RFI}_{\mathrm{P}}=$ mean $+\mathrm{CGP}+\mathrm{SB}+$ Animal + Residual;

$\mathrm{RFI}_{\mathrm{M}}=$ mean + Animal + Residual.

where CGP is the contemporary group of test year and group combination; $S B$ is sire breed (Angus, Charolais or Hybrid); SAGE is the slaughter age in days; the subscript $\mathrm{P}$ and $\mathrm{M}$ represent the phenotype and marker score respectively. Animal is assumed as a random effect in the above models (Animal $\sim N\left(0, A \sigma_{a}^{2}\right)$ ), where $A$ is a matrix of additive genetic relationships among animals and $\sigma_{a}^{2}$ is the additive genetic variance for the trait under consideration. Residual is assumed as a random effect in the above models (Residual $\sim N\left(0, I \sigma_{e}^{2}\right)$ ), where $I$ is an identity matrix and $\sigma_{e}^{2}$ is the residual variance for the trait under concern.

\subsection{Evaluation for the selection efficiency of MABLUP}

The selection efficiency of MABLUP was evaluated by the prediction accuracy, which was calculated as

$$
\text { Accu }=\sqrt{\left(1-\frac{S_{i}^{2}}{\left(1+f_{i} \sigma_{A}^{2}\right)}\right)},
$$

where $S_{i}^{2}$ is the estimated standard error of individual $i ; f_{i}$ is the inbreeding coefficient of animals; $i$ and $\sigma_{A}^{2}$ are the additive genetic variance [17].

\section{Results}

\subsection{Results of the association test}

The variance components of BayesB are summarized in Table 2. The basic descriptive statistics for all significant $(P$ $<0.10)$ SNP marker obtained from the linear model for MAS are summarized in Table 3. The numbers of significant SNP marker for CWT, BF, REA, GDF, LMY and RFI were 75, 54, 67, 57, 44 and 50, respectively. The contribution of the significant SNPs marker to the phenotypic variance is summarized in Table 4.

\subsection{Genetic parameters for marker scores and pheno- types}

Table 5 lists the heritability and genetic correlation of

Table 2 The phenotypic and genetic variance for carcass weight (CWT), carcass back fat (BF), carcass rib eye area (REA), carcass grade fat (GDF), lean meat yield (LMY), and residual feed intake (RFI)

\begin{tabular}{lcc}
\hline Trait & Genetic variance & Total variance \\
\hline CWT & 337.511 & 693.063 \\
BF & 3.635 & 14.004 \\
REA & 21.179 & 58.221 \\
GDF & 3.875 & 13.916 \\
LMY & 2.855 & 12.019 \\
RFI & 0.165 & 0.796 \\
\hline
\end{tabular}


Table 3 The descriptive statistics of markers to calculate marker score for carcass weight (CWT), carcass back fat (BF), carcass rib eye area (REA), carcass grade fat (GDF), lean meat yield (LMY), and residual feed intake (RFI)

\begin{tabular}{lrrrrc}
\hline Trait & N & Mean & Min & Max & Std Dev \\
\hline CWT & 75 & 23.04 & -34.97 & 107.50 & 19.06 \\
BF & 54 & 0.20 & -9.70 & 8.61 & 2.57 \\
REA & 67 & 2.77 & -12.11 & 21.75 & 5.15 \\
GDF & 57 & -6.69 & -13.76 & 7.33 & 3.21 \\
LMY & 44 & -0.85 & -6.04 & 5.01 & 1.78 \\
RFI & 50 & 0.40 & -0.97 & 1.70 & 0.45 \\
\hline
\end{tabular}

Table 4 The contribution of the significant SNPs marker to the phenotypic variance for carcass weight (CWT), carcass back fat (BF), carcass rib eye area (REA), carcass grade fat (GDF), lean meat yield (LMY), and residual feed intake (RFI)

\begin{tabular}{lcccc}
\hline Trait & $N$ & $\begin{array}{c}\text { Variance of the } \\
\text { significant markers }\end{array}$ & $\begin{array}{c}\text { Phenotypic } \\
\text { variance }\end{array}$ & Contribution \\
\hline CWT & 75 & 102.8 & 693.063 & 0.148 \\
BF & 54 & 2.21 & 14.004 & 0.158 \\
REA & 67 & 8.49 & 58.221 & 0.146 \\
GDF & 57 & 2.05 & 13.916 & 0.147 \\
LMY & 44 & 1.43 & 12.019 & 0.119 \\
RFI & 50 & 0.095 & 0.796 & 0.119 \\
\hline
\end{tabular}

Table 5 The heritability and genetic correlation of marker score and phenotype for carcass weight (CWT), carcass back fat (BF), carcass rib eye area (REA), carcass grade fat (GDF), lean meat yield (LMY), and residual feed intake (RFI)

\begin{tabular}{lcc}
\hline Trait & Phenotype & Marker score \\
\hline CWT & $0.36 \pm 0.10^{\mathrm{a}}$ & \\
& $0.75 \pm 0.08^{\mathrm{b})}$ & $0.55 \pm 0.10$ \\
BF & $0.31 \pm 0.10$ & \\
& $0.69 \pm 0.09$ & $0.79 \pm 0.10$ \\
REA & $0.31 \pm 0.08$ & \\
& $0.87 \pm 0.06$ & $0.79 \pm 0.10$ \\
GDF & $0.35 \pm 0.10$ & \\
& $0.77 \pm 0.08$ & $0.89 \pm 0.10$ \\
LMY & $0.27 \pm 0.09$ & $0.74 \pm 0.11$ \\
& $0.78 \pm 0.09$ & \\
RFI & $0.32 \pm 0.09$ & $0.69 \pm 0.10$ \\
& $0.85 \pm 0.07$ &
\end{tabular}

a) The diagonal elements of each trait row are heritability. b) The nondiagonal elements of each trait row are genetic correlation.

marker score and phenotype for CWT, BF, REA, GDF, LMY and RFI. The heritability of marker score for RFI and five $\mathrm{CM}$ traits were very high because the environmental influences were expected to be minimal, which were different from typical production traits $[7,8]$. The genetic correlations of marker score and phenotype for CWT, BF, REA, GDF, LMY and RFI were $0.75,0.69,0.87,0.77,0.78$, and 0.85 , respectively.

\subsection{Prediction accuracy for MABLUP and CBLUP}

The detailed results are shown in Figure 1. Compared with CBLUP, MABLUP obviously improved the prediction accuracies of phenotypic EBV for CWT, BF, GDF, REA, LMY and RFI. The average prediction accuracies of phenotypic EBV for CWT, BF, GDF, REA, LMY and RFI were increased by $0.05,0.16,0.24,0.23,0.17$ and 0.19 , respectively.

\section{Discussion}

\subsection{Factors affecting the model}

A comparison can be made with the results from Tang et al. [9], where a total of 2632 animals genotyped by 233 markers, and the average MABLUP EBV accuracy obtained from their study was 0.55 . Although there were fewer animals in our study, the denser SNP markers still provided a higher MABLUP accuracy (0.76). It means that highdensity SNP markers can result in high selection accuracy because LD between QTLs and markers is stronger in a high density marker panel than that in lower density panels, and it is expected the high density maker panel be able to capture more QTLs than the lower one that was used by Tang et al. [9]. The significance test and estimation of marker effect is the key to the success of the development of marker-assisted evaluation model in commercial livestock population [18]. In this study, the number of SNP markers was much larger than the number of animals; therefore, a Bayesian method was employed to simultaneously estimate the marker effects and variance [3]. BayesC was first implemented to estimate the variance of genetic and residual, respectively, and then, a reasonable $\pi$ value from BayesCpi was used in the BayesB model to estimate the effect and variance for each marker. However, BayesB can only estimate the variances and effects of all markers, but cannot test

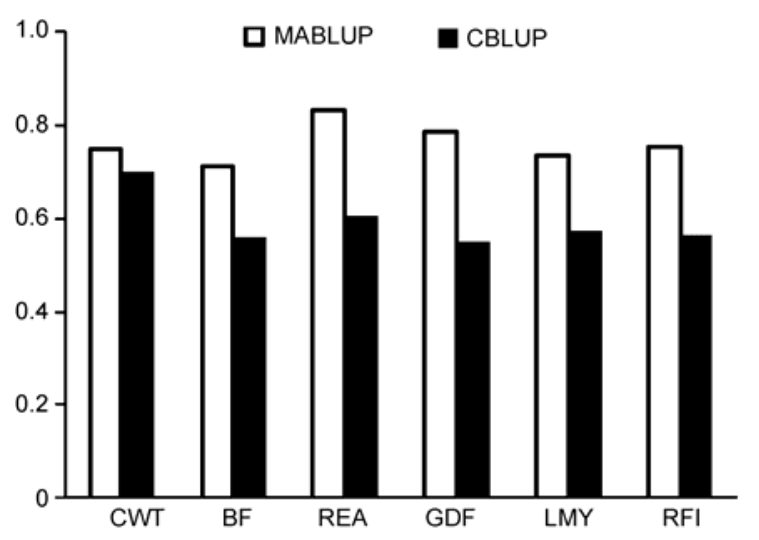

Figure 1 Accuracy of marker assisted BLUP (MABLUP) and conventional BLUP (CBLUP) for carcass weight (CWT), carcass back fat (BF), carcass rib eye area (REA), carcass grade fat (GDF), lean meat yield (LMY), and residual feed intake (RFI). 
the significance of them. Therefore, for choosing a reasonable subset of markers to develop the MABLUP model, three marker sets (200, 400 and 800 SNPs) were selected first based on the size of variance resulting from the BayesB model. Then, a mutiple-marker regression model was implemented in these marker sets to detect the significance of markers. The results showed that the marker set with 200 SNPs was a reasonable choice because of the following two reasons: (i) the variance of the 200 SNPs obtained from BayesB explained most of the genetic variance of all of the six traits; (ii) the markers from the 200 multi-marker analysis were easy to converge for most of the six traits in the subsequent two-trait MAS model analysis. The average genetic correlation between the marker score of the significant markers and phenotype was 0.79 , showing that this 200 marker set was a good choice after a strict examination of all the markers in this study.

\subsection{Implementation of the MABLUP model}

A major limitation in applying this model for carcass traits was that phenotypic data could only be obtained after slaughtering the animal, as the breeding opportunity for the next generation is lost. One solution is to use indicator traits [8], where intramuscular fat (IMF) measured by ultrasound and molecular breeding value (MBV) for marbling were discussed as the indicators for marbling score (MRB) of slaughtered steers. As these authors pointed out, using ultrasound IMF as the indicator was still not sufficient enough to obtain a reasonable genetic gain for IMF, and the work of progeny testing and sib testing for IMF needs to continue. An alternative is to use frozen semen with artificial insemination (AI) from the best animals, by storing sufficient semen from all candidates prior to slaughter and the measurement of the trait. However, in most beef cattle operations, AI is not a popular practice. In summary, MABLUP for carcass traits should be considered as an alternative choice to achieve highly accurate EBVs compared with CBLUP.

Interestingly, MABLUP fits RFI particularly well. Beef cattle can still breed after RFI data collection. However, Tang et al. [9] pointed out that for MABLUP applications that the marker scores need to be re-evaluated in different populations to ensure the LD phases of marker and QTL. The marker score also needs to be re-evaluated in each subsequent generation. Sherman et al. [19] reported six SNPs, which were found to explain $6.9 \%$ of the phenotypic variation of RFI. Unfortunately, these SNPs were not included in our study because these markers did not meet the criteria for marker quality in our analysis. The accuracy may be higher if we include these markers since they were verified in this population.

\subsection{Efficiency of genomic selection in beef cattle popu- lation}

The initial objective of this study was to investigate the fea- sibility of genomic selection in the beef cattle population at the University of Alberta. The reference population with 604 animals from the first 4 years (2003-2006) was used to calculate marker effects; the rest of the 318 animals of the last 2 years (2007-2008) were used as a validation population. The correlations between GEBV and EBV for CWT, BF, GDF, LMY, REA and RFI in the validation population were $0.24,0.08,0.32,0.22,0.2$, and 0.32 , respectively (Table 6). Unfortunately, the results showed that using the prediction equation developed from the reference population to predict the GEBV in the validation population did not work well as evidenced by the low average correlation between GEBV and EBV for all six traits (0.24). The accuracy of GEBV depends on four factors [20,21]: (i) the level of linkage disequilibrium (LD) between the markers and the QTL; (ii) the number of animals with phenotypes and genotypes in the reference population; (iii) the heritability of the trait; and (iv) the distribution of QTL effects. To overcome the first two factors, a denser marker panel may be needed, as well as accumulating more phenotypic and genotypic records for the reference population. Another reason for reduced accuracy of genomic selection was the number of breeds. Harris et al. [22] reported that SNP estimates calculated from a Holstein-Friesian reference population did not produce accurate genome-wide marker scores in Jersey bulls and vice versa. The population in this study was a beef composite population that was a crossbred of many beef and dairy breeds as described by Goonewardene et al. [23]. A breed specific or higher density marker panels should be investigated for genomic selection in future.

\section{Conclusion}

The two-trait marker-assisted evaluation model increased the estimation accuracy of EBV of carcass traits and RFI. It is therefore a potential alternative to use genome-wide MAS instead of GS in beef cattle breeding programs for genetic improvement of $\mathrm{CM}$ and RFI traits at least until a large enough database of phenotypes and genotypes is available for feasible genomic selection.

Table 6 Accuracy of genomic selection for carcass weight (CWT), carcass back fat (BF), carcass rib eye area (REA), carcass grade fat (GDF), lean meat yield (LMY), and residual feed intake (RFI)

\begin{tabular}{lc}
\hline Trait & Correlation between GEBV and EBV \\
\hline CWT & 0.23663 \\
BF & 0.07961 \\
REA & 0.31739 \\
GDF & 0.21897 \\
LMY & 0.19899 \\
RFI & 0.3178 \\
\hline
\end{tabular}


This work was supported by the Alberta Livestock and Meat Agency (ALMA) (G550000101), ALMA/Alberta Agricultural Research Institute (AARI) (G550000127), and National Natural Science Foundation of China (30771534) who are greatly acknowledged.

1 Crews D H Jr. Genetics of efficient feed utilization and national cattle evaluation: A review. Genet Mol Res, 2005, 4: 152-165

2 Dekkers J C M. The use of molecular genetics in the improvement of agricultural populations. Nat Rev Genet, 2002, 3: 22-32

3 Meuwissen T, Hayes B J, Goddard M. Prediction of total genetic value using genome-wide dense marker maps. Genetics, 2001, 157: 1819-1829

4 Goddard E M, Hayes J B. Mapping genes for complex traits in domestic animals and their use in breeding programmes. Nat Rev Genet, 2009, 10: 381-391

5 van Raden P M, Van Tassell C P, Wiggans G R, et al. Invited review: Reliability of genomic predictions for North American Holstein bulls. J Dairy Sci, 2009, 92: 16-24

6 Hayes B, Bowman P, Chamberlain A, et al. Accuracy of genomic breeding values in multi-breed dairy cattle populations. Genet Sel Evol, 2009, 41: 51

7 Kachman S D. Incorporation of marker scores into national genetic evaluations. Proc of the 9th genetic prediction workshop, Beef improment federation, Kansas City, Mo, 2008. 92

8 MacNeil M, Nkrumah J, Woodward B, et al. Genetic evaluation of Angus cattle for carcass marbling using ultrasound and genomic indicators. J Anim Sci, 2010, 88: 517-522

9 Tang G Q, Li X W, Wang Z Q. Developing marker-assisted models for evaluating growth traits in Canadian beef cattle genetic improvement. Livest Sci, 2011, 138: 1-3

10 Olfert E D, Cross B M, McWilliam A A. Guide to the care and use of experimental animals. Vol. 1. Ottawa, Ontario, Canada: Canadian Council on Animal Care, 1993

11 Nkrumah J, Wang Z, Moore S S. Primary genome scan to identify putative quantitative trait loci for feedlot growth rate, feed intake, and feed efficiency of beef cattle. J Anim Sci, 2007, 85: 3170-3181
12 Basarab J A, Price M A, Aalhus J L, et al. Residual feed intake and body composition in young growing cattle. Can J Anim Sci, 2003, 83: 189-204

13 Hayes B J, Bowman P J, Chamberlain A J, et al. Genomic selection in dairy cattle: Progress and challenges. J Dairy Sci, 2009, 92: 433-443

14 Fernando R, Garrick D. GenSel-User manual for a portfolio of genomic selection related analyses. Animal Breeding and Genetics, Iowa State Univ, Ames, 2008

15 Madsen P, Jensen J. A user's guide to DMU. A package for analyzing multivariate mixed models. Version 6, release 4.6. Danish Institute of Agricultural Sciences, 2006. 27

16 Garrick D. The nature and scope of some whole genome analyses in US beef cattle. In: Proceedings of 41st Beef Improvement Federation Annual Research Symposium: 2009. Sacramento, USA. 2009, 92: 102

17 Gilmour A R, Gogel B J, Cullis B R, et al. ASReml user guide release 3.0. VSN International Ltd., Hemel Hempstead, HP1 1ES, UK. http://www.vsni.co.uk, 2009. 275

18 Dekkers J C M. Commercial application of marker- and gene-assisted selection in livestock: Strategies and lessons. J Anim Sci, 2004, 82(13 suppl): E313-E328

19 Sherman E L, Nkrumah J D, Murdoch B M, et al. Identification of polymorphisms influencing feed intake and efficiency in beef cattle. Anim Genet, 2008, 39: 225-231

20 Hayes B, Goddard M. Technical note: Prediction of breeding values using marker-derived relationship matrices. J Anim Sci, 2008, 86: 2089-2092

21 Goddard M. Genomic selection: Prediction of accuracy and maximisation of long term response. Genetica, 2009, 136: 245-257

22 Harris B L, Johnson D L, Spelman R J, et al. Genomic selection in New Zealand and the implications for national genetic evaluation. Proc. 36th International Committee for Animal Recording (ICAR) Conference, Niagara Falls, NY, 2009. 325-330

23 Goonewardene L A, Wang Z, Price M A, et al. Effect of udder type and calving assistance on weaning traits of beef and dairy $\mathrm{X}$ beef calves. Livest Prod Sci, 2003, 81: 47-56

Open Access This article is distributed under the terms of the Creative Commons Attribution License which permits any use, distribution, and reproduction in any medium, provided the original author(s) and source are credited. 